\title{
Percolation model of combustion
}

\author{
D. Rybakov ${ }^{l, *}$, Kh. Lamazhapov ${ }^{l}$ \\ ${ }^{1}$ Samara state transport university, Russian Federation
}

\begin{abstract}
Strong dependencies in equations cause dramatic boost of reaction rates in some rare but meaningful areas of a combustion gap. That leads to intermittent behavior of some values and for these reasons averaging approaches in calculations are not relevant. We use percolation theory instead in order to explain ignition delay and completeness of combustion.
\end{abstract}

In ideal conditions reaction rate depends on molecular concentration and some factor $\mathrm{k}$ as

$$
-\mathrm{da}_{1} / \mathrm{dt}=-\mathrm{da}_{2} / \mathrm{dt}=\mathrm{k} \mathrm{a}_{1} \mathrm{a}_{2}
$$

Here the factor $\mathrm{k}$ depends on temperature as

$$
\mathrm{k}=\mathrm{k}_{0} \mathrm{e}^{-E R^{-1} \mathrm{~T}^{-1}}
$$

and apparently this coefficient has a remarkable variation range. Assume typical activation energy $\mathrm{E}$ equals $40 \mathrm{kcal} \cdot \mathrm{mol}^{-1}$, the universal gas constant $\mathrm{R}$ equals $1.99 \mathrm{kcal} \cdot \mathrm{mol}^{-1} \cdot \mathrm{K}^{-1}$ and temperature $\mathrm{T}$ ranges over 500 to 1000 Kelvin. Then here we observe $\mathrm{k}(500)=10^{-17.4}$ and $\mathrm{k}(1000)=10^{-8.7}$ so

$$
\mathrm{k}(1000) / \mathrm{k}(500) \approx 10^{-8.7}
$$

So if temperature $\mathrm{T}$ increases 2 times the coefficient $\mathrm{k}(\mathrm{T})$ will increase billion times! [1]

Molecular concentrations $\mathrm{a}_{1}, \mathrm{a}_{2}$, temperature $\mathrm{T}$ and other magnitudes are statistically distributed in real life. Also, temperature has a feedback coupling with local energy output. Therefore, values form random fields in a combustion gap. Stochastic behavior leads to dramatic intensification of combustion processes in some rare but meaningful areas. Moreover, one can investigate intermittent random fields using statistical moments applying the following rule: if $\mathrm{M}_{\mathrm{i}}$ increases as $\mathrm{i}$ increases this will point out irrelevancy of averaging approaches [2].

$$
\mathrm{M}_{\mathrm{i}}=\sqrt[i]{\int_{0}^{\infty}(\mathrm{k}(\mathrm{x}))^{\mathrm{i}} \cdot \frac{\mathrm{e}^{-(\mu-\mathrm{x})^{2} / 2 \sigma^{2}}}{\sigma \sqrt{2 \pi}} \mathrm{dx}}
$$

The root sign is used in order to keep physical unit consistency. Numerical calculation for $\mathrm{T} \sim \mathrm{N}(\mu=100, \sigma=100)$ yields the following moments: $\mathrm{M}_{1}=8 \cdot 10^{-9}, \mathrm{M}_{6}=8 \cdot 10^{-7}, \mathrm{M}_{12}=8 \cdot 10^{-6}$.

For instance, a fuel spray notably clear demonstrates intermittent properties because it definitely encapsulates pure fuel droplets and areas of irregularly saturated vapor. There exists a set of small areas giving a boost to local burning process. These areas configure clusters with very irregular structure and could be described by fractal dimension D. So in

\footnotetext{
*Corresponding author: dim2r@yandex.ru
} 
some cases inflaming front should pass a very long path in between different areas with very different properties. So some physical properties directly depend on irregular geometry of different areas. And geometry depends on active element volume fraction $\rho$. In our article, value $\rho$ takes into account element intersections and represents final volume fraction. Critical value could be obtained analytically as well as numerically $\rho_{c}=0.29[4]$. We performed 3D computer simulation over percolation system with soft-core spherical elements in order to find out some properties and features of clusters and surfaces important for combustion theory (figure 1). Model includes a set of randomly located spheres such that a single sphere represents a fuel droplet surrounded by fuel vapor. We assume that ignition and flame propagation is possible only within spheres and clusters.

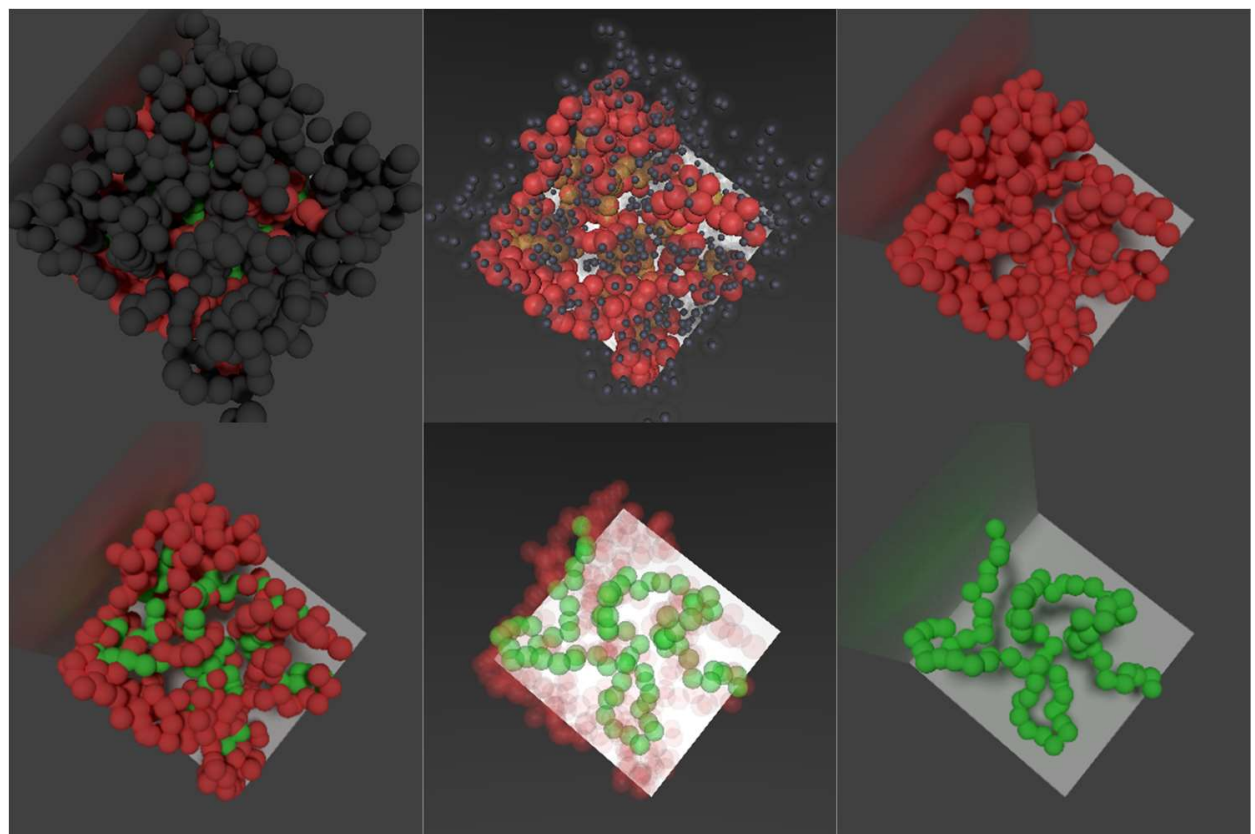

Fig. 1. Different representations of the same scene including a droplet cluster. The cluster is red. The path inside the cluster is green. Orphan droplets are black.

Computer simulation shows that flame propagation time $t_{\text {prop }}(\rho)$ from one point to another depends on the shortest path length between two points $L_{\text {path }}(\rho)$. The length significantly increases at the percolation threshold and exceeds size of a gap L. So lengths of paths explain an ignition timeout and its statistical properties (figure 2). 

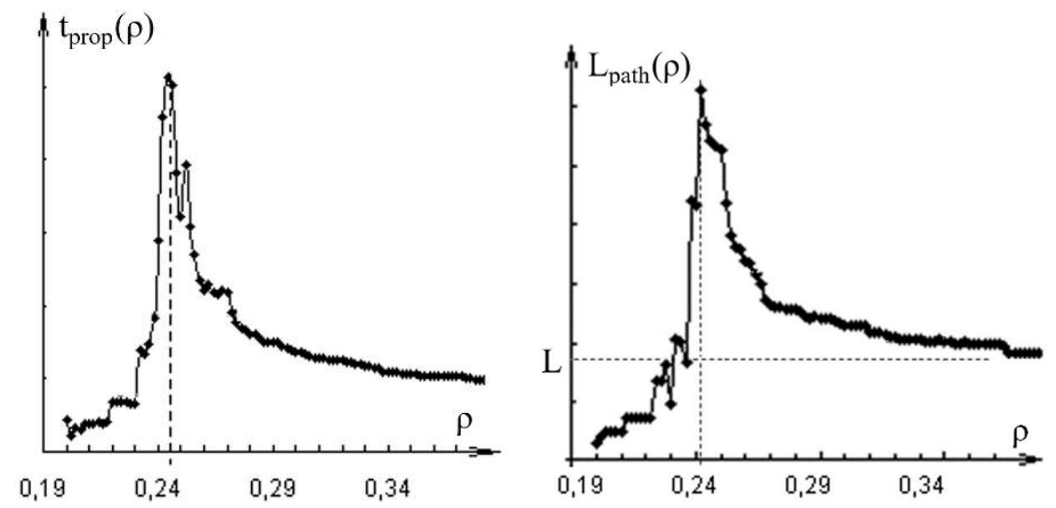

Fig. 2. Flame propagation time vs path length inside a cluster.

At the beginning stage a flame propagates beneath a cluster surface. The closer elements located the smaller total surface is available from outside a cluster. We calculated available surface fraction as

$$
F(\rho)=\sum s_{i} / \sum S_{i}
$$

Here $\mathrm{S}_{\mathrm{i}}=4 \pi \mathrm{r}^{2}$ is total element surface and $\mathrm{s}_{\mathrm{i}}$ is external fraction of element surface. Ball size variation within an ensemble has moderate influence on external surface fraction (figure 2).

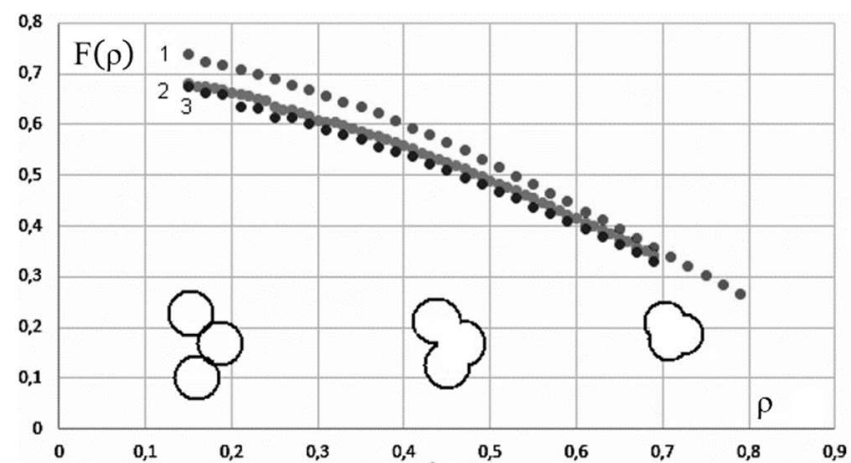

Fig. 3. Cluster surface relative to total surface of elements. 1) Balls have equal size.

2) Ball radiuses are statistically distributed $R_{\max } / R_{\min }=5$

3) Ball radiuses are statistically distributed $R_{\max } / R_{\min }=10$

Also in some cases path does not exist between two points. That means that some areas are inaccessible by the flame. So completeness of combustion $\mathrm{C}$ is attributable to a balance of accessible and inaccessible areas in a percolation system.

$$
\mathrm{C}(\rho)=\mathrm{N}_{\text {cluster }} / \mathrm{N}_{\text {total }}
$$

At the best case ignition starts from an element belonging to a biggest cluster. Thus completeness of combustion does not exceed a fraction of the biggest cluster in the whole system (figure 4). 


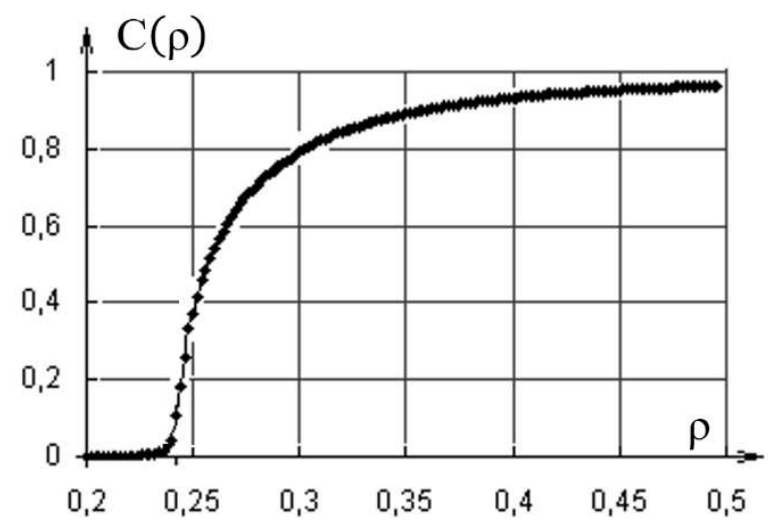

Fig. 4. Relative number of element in the biggest cluster. Completeness of combustion at the initial stage does not exceed that fraction.

\section{References}

1. Ya. B. Zel'dovich, G.I. Barenblatt, V.B. Librovich, G.M.Mahviladze, Mathematical theory of combustion and explosion. Science, (1980)

2. Ya. B. Zel'dovich, S. A. Molchanov, A. A. Ruzmaikin, and D. D. Sokolov, Intermittency in random media. Sov. Phys. Usp., (1987)

3. M. Sahimi, Applications of percolation theory. London : Taylor \& Francis, (1992)

4. I. Balberg, C. H. Anderson, S. Alexander and N. Wagner, Excluded volume and its relation to the onset of percolation. Phys. Rev. B, 30, 7, (1984) 